\title{
Aplicação da Internet das Coisas para Desenvolvimento de Ferramenta de Apoio no Monitoramento Sistemático da Umidade do Solo
}

\author{
Luiz Paulo Santos ${ }^{1}$, Daiene F. S. Corado ${ }^{1}$, Humberto X. de Araújo ${ }^{1}$ \\ ${ }^{1}$ Universidade Federal do Tocantins (UFT) \\ Palmas - TO. \\ \{luiz.santos, daiene, hxaraujo\}@uft.edu.br
}

\begin{abstract}
Identify the soil moisture and a determining factor to propitiate satisfactory conditions for the development of any vegetative vegetation system. An automated irrigation control system, based on the potential of ground water, is a tool that can optimize the use of natural resources in water. This article aims to present a prototype based on the Internet of Things (IoT) for the development of a tool to support systematic soil moisture monitoring of the vegetation of the Environmental Studies Unit (NEAMB), linked to the Biology Sciences course of the Federal University of Tocantins (UFT), Porto Nacional campus. The development of the prototype aims to offer a low cost automation for the irrigation process, above all, to provide a database with continuous information on the characteristics of the soil. was used in this work an ESP8266 microcontroller, together with sensors and actuators that allow to do both the control of the irrigation system and the real-time monitoring of soil moisture characteristics. The use of this tool broadens the horizons of the researches developed as researchers in the area can achieve a better understanding of the impacts of changes in soil moisture on the development of the vegetative systems surveyed
\end{abstract}

Resumo. Identificar a umidade do solo é um fator determinante para propiciar condições satisfatórias ao desenvolvimento de qualquer sistema vegetativo cultivado. Um sistema automatizado de controle de irrigação, com base no potencial de água no solo, é uma ferramenta que pode otimizar a utilização racional de recursos hídricos. Este artigo visa apresentar um protótipo baseado em Internet das Coisas (IoT) para o desenvolvimento de uma ferramenta de apoio às pesquisas em monitoramento sistemático de umidade do solo da casa de vegetação do Núcleo de Estudos Ambientais (NEAMB), vinculado ao curso de Ciências Biológicas da Universidade Federal do Tocantins (UFT), campus de Porto Nacional. O desenvolvimento do protótipo visa, além de oferecer uma automatização de baixo custo para o processo de irrigação, sobretudo, entregar uma base de dados com informações contínuas das características do solo. Foram utilizados neste trabalho um microcontrolador ESP8266, juntamente com sensores e atuadores que permitam fazer tanto o controle do sistema de irrigação quanto o monitoramento em tempo real das características de umidade do solo. $O$ uso desta ferramenta amplia os horizontes das pesquisas desenvolvidas na casa de vegetação, uma vez que os pesquisadores da área podem alcançar uma maior compreensão dos impactos das alterações de umidade do solo no desenvolvimento dos sistemas vegetativos pesquisados. 


\section{Introdução}

No modelo de sociedade contemporâneo há uma crescente busca por aliar as tecnologias existentes à automação das diversas atividades rotineiras do cotidiano. O conceito de Internet das Coisas surgiu com a popularização da internet e seu principal objetivo é interligar os equipamentos que usamos no dia a dia com a internet [de Oliveira 2017]. O termo Internet das Coisas deriva do inglês, Internet of Things (IoT) é definido por [Santos et al. 2016] como uma extensão da internet atual, que busca proporcionar aos objetos do uso cotidiano com capacidade computacional e comunicativa, sejam eles quais forem, a conexão com a internet. A conectividade com a rede mundial de computadores torna possível o controle remoto dos objetos, bem como viabiliza o acesso destes como provedores de serviços, gerando grandes oportunidades de aplicação, em âmbito tanto acadêmico quanto industrial. O avanço no uso da IoT é veloz e, em grande parte, se deve ao barateamento das tecnologias envolvidas no processo de produção de objetos conectados. Atualmente, a relação de preço-recursos dos elementos necessários para a disseminação da IoT tornou-se factível, e propiciou uma aceleração na utilização desses objetos [de Oliveira 2017]. Dados da Gartner, empresa de análise acerca do mercado de Tecnologia da Informação (TI), apontam que até 2020 mais de 26 milhões de dispositivos estarão conectados a alguma "coisa" [Morgan 2018] . Na Tabela 1 pode-se verificar as principais tecnologias que propiciaram o desenvolvimento e possibilitaram a ampliação da utilização da Internet das Coisas. Neste trabalho foi enfatizado, dentre as tecnologias existentes para a exploração da IoT, o microcontrolador ESP8266, produzido pela fabricante Espressif, uma vez que este foi utilizado no desenvolvimento do protótipo proposto. Pode-se observar hodiernamente o esforço global com as questões de preservação ambiental. Seguindo esta linha, a pesquisa em questão visa aplicar o uso de internet das coisas no controle de análise do solo e de irrigação consciente dos sistemas vegetativos.

Table 1. Evolução das tecnologias associadas ao conceito IoT.

\begin{tabular}{|l||l|}
\hline TECNOLOGIA & DESCRIÇÃO \\
\hline RFID & $\begin{array}{l}\text { A tecnologia de identificação por radiofrequência - Radio Frequency Identifi- } \\
\text { cation (RFID) - surgiu em 1940 e trata-se de um equipamento que envia, por } \\
\text { radiofrequência uma identificação única. }\end{array}$ \\
\hline RSSF & $\begin{array}{l}\text { Redes de Sensores Sem Fio (RSSF) são redes compostas de dezenas, centenas } \\
\text { ou até milhares de nós microprocessados, com capacidade de comunicação } \\
\text { sem fio e alimentados por baterias. }\end{array}$ \\
\hline $\begin{array}{l}\text { REDES DE } \\
\text { REMPUTADO- }\end{array}$ & $\begin{array}{l}\text { Popularização das tecnologias de comunicação, através da Internet, usando os } \\
\text { protocolos da família TCP/IP e, posteriormente, por meio das redes WIFI, que } \\
\text { tornaram possível a mobilidade, dispensando o uso de fios para a interligação } \\
\text { de dispositivos próximos. }\end{array}$ \\
\hline $\begin{array}{l}\text { DISPOSITIVOS } \\
\text { PORTÁTEIS }\end{array}$ & $\begin{array}{l}\text { A mobilidade proporcionada pelos dispositivos portáteis, como notebooks, } \\
\text { tablets e, em especial, os smartphones tornou mais fácil e desejável acessar as } \\
\text { informações e os equipamentos de qualquer parte. }\end{array}$ \\
\hline $\begin{array}{l}\text { MICROCONTRO- } \\
\text { LADORES }\end{array}$ & $\begin{array}{l}\text { Microcontroladores atualmente possuem diversas funcionalidades agregadas, } \\
\text { além de simplesmente interfaces de entrada e saída (I/O), como memória } \\
\text { RAM, memória EPROM para programas e dados e circuito de oscilador } \\
\text { (clock), interfaces de comunicação (serial, USB) interfaces de rede, ethernet, } \\
\text { WiFi e Bluetooth, o que possibilita um número de aplicações sem preceden- } \\
\text { tes. }\end{array}$ \\
\hline
\end{tabular}


A execução da irrigação com base no potencial de água no solo é a maneira mais racional de uso da água e o processo de irrigação manual é uma barreira a ser transposta, uma vez que nesse tipo de método a irrigação é irregular, o que pode acarretar tanto a irrigação excessiva do solo, levando à perda de nutrientes do solo pelo processo de lixiviação, quanto a irrigação deficiente, permitindo que se esgote grande parte da água disponível armazenada entre uma irrigação e outra [Júnior et al. 2009], [Souza et al. 2002]. As pesquisas realizadas por [Júnior et al. 2009] e [dos Santos and Pereira 2010] também apontam que técnicas de controle de irrigação devem ser aplicadas levando-se em consideração fatores como: 1) Atributos físico-hídricos do solo. 2) Fisiologia da planta. 3) Cômputo do balance de água na zona radicular. 4) Modelos climatológicos. Ao se pensar em um ambiente acadêmico, a implantação de uma ferramenta automatizada de monitoramento de irrigação amplia consideravelmente os horizontes das pesquisas. Dispor de uma base de dados onde é possível armazenar características do solo medidas periodicamente, como umidade e temperatura, permite aos pesquisadores realizarem análises e experimentos com uma maior eficiência. Mesmo existindo alguns bancos de dados de grande porte, pode-se verificar que as observações sistemáticas da umidade do solo ainda são escassas, especialmente porque grande parte das técnicas existentes para este tipo de averiguação ainda possuem um custo muito elevado [Menezes et al. 2013]. Diante de todo o exposto acima, o objetivo do desenvolvimento da ferramenta de monitoramento sistemático da umidade do solo é, não somente oferecer uma automatização para o processo de irrigação em si, mas, principalmente, monitorar e disponibilizar uma base de dados relativos às características do solo ao longo do tempo, uma vez que, munidos de medidas de umidade do solo contínuas e consistentes, pesquisadores da área podem alcançar uma maior compreensão dos impactos das alterações da umidade do solo no desenvolvimento dos diversos sistemas vegetativos pesquisados.

\section{Materiais e Métodos}

O desenvolvimento deste protótipo foi pensado para automatização da casa de vegetação do Núcleo de Estudos Ambientais (NEAMB), vinculado ao curso de Ciências Biológicas da Universidade Federal do Tocantins, campus de Porto Nacional. A Figura 1 mostra a estrutura atual da referida casa, onde pode-se observar o quanto sua composição é simplista, sendo utilizada quase que somente para a produção de mudas. O protótipo visa oferecer melhores condições para o desenvolvimento de pesquisas do NEAMB, fazendo com que se explore de maneira mais eficiente este rico espaço.

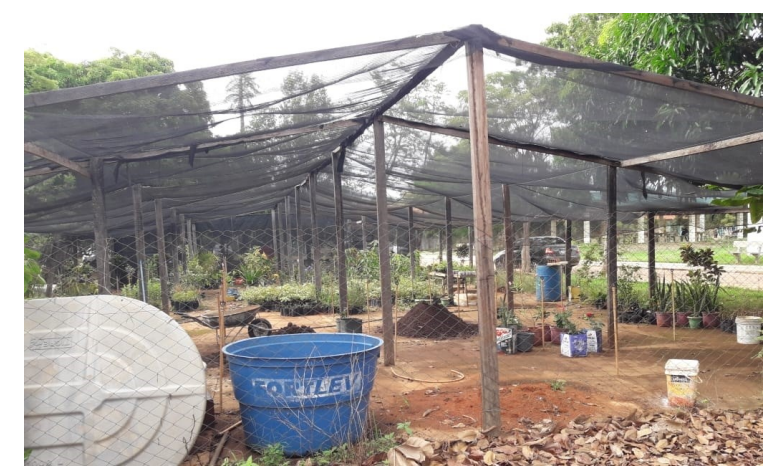

Figura 1. Casa de Vegetação do NEAMB 
Valer-se de um sistema automatizado de irrigação é vantajoso tanto pela questão do consumo consciente dos recursos hídricos, uma vez que o sistema de agoamento é acionado somente quando necessário, quanto pela questão da observância das características dos sistemas vegetativos cultivados, já que é possível realizar a calibragem dos sensores do equipamento de acordo com a umidade e temperatura mais favorável para diferentes sistemas que possam estar sendo cultivados na casa de vegetação. Abaixo serão apresentados os procedimentos e materiais utilizados para o desenvolvimento do protótipo.

\section{- Materiais}

1. Módulo wifi ESP8266 - este módulo é uma placa de desenvolvimento que combina o chip ESP8266, uma interface usb-serial e um regulador de tensão 3.3V, conforme apresenta a Figura 2. Para a programação deste microcontrolador foi utilizada a IDE do arduíno, com comunicação via cabo micro-usb.

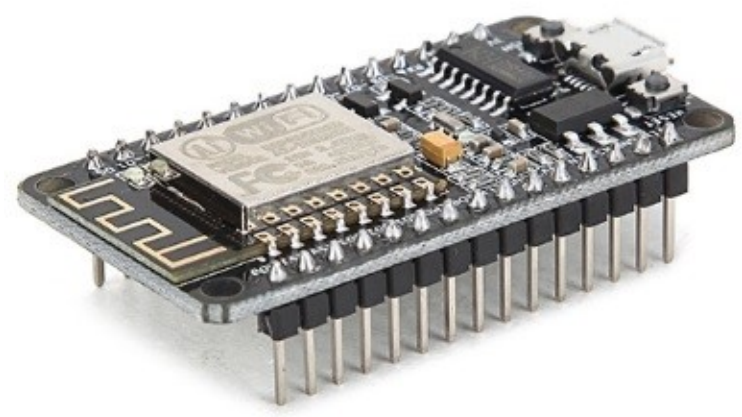

Figura 2. Módulo wifi ESP8266

2. Sensor de umidade e temperatura DHT11 - este sensor de temperatura e umidade permite leituras de temperaturas entre $0{ }^{\circ} \mathrm{C}$ e $50{ }^{\circ} \mathrm{C}$ e umidade entre $20 \%$ a $90 \%$, conforme apresenta a Figura 3.

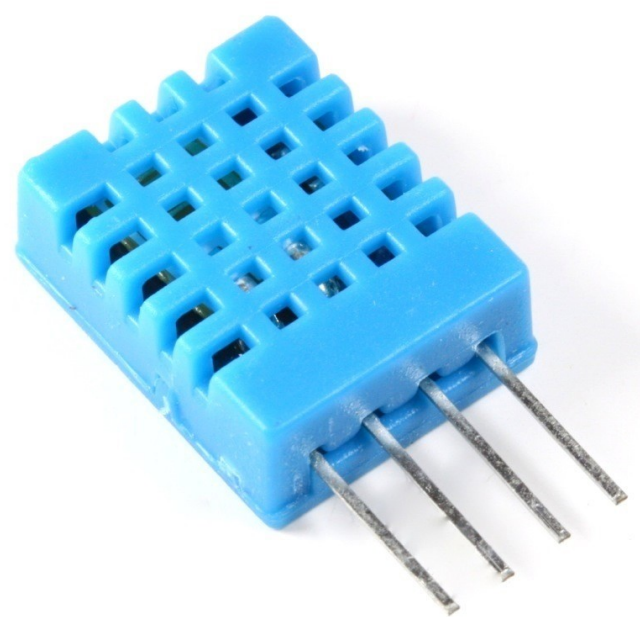

Figura 3. Sensor de umidade e temperatura DHT11 
3. Módulo Relé - Utilizado para o acionamento de cargas elétricas, ou seja, pode controlar equipamentos eletrônicos, desde que a corrente dos dispositivos a serem controladas não ultrapasse $10 \mathrm{~A}$ (ampéres), conforme apresenta a Figura 4.

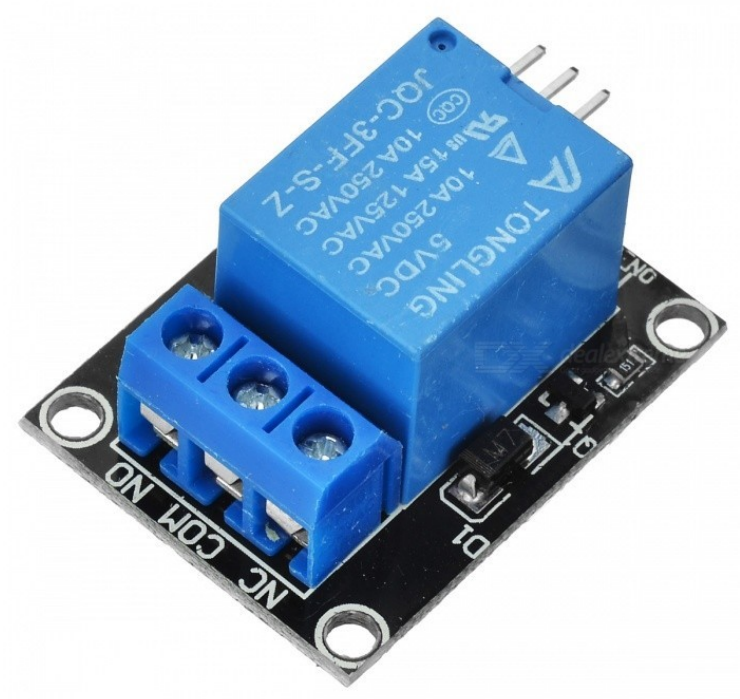

Figura 4. Módulo Relé

4. Bomba - Para a simulação da irrigação foi usado o modelo Moto Bomba SB 1000 produzido pela empresa Sarlo. Essa bomba é utilizada para a circulação de água em aquários, sendo uma bomba de grande capacidade, conforme apresenta a Figura 5.

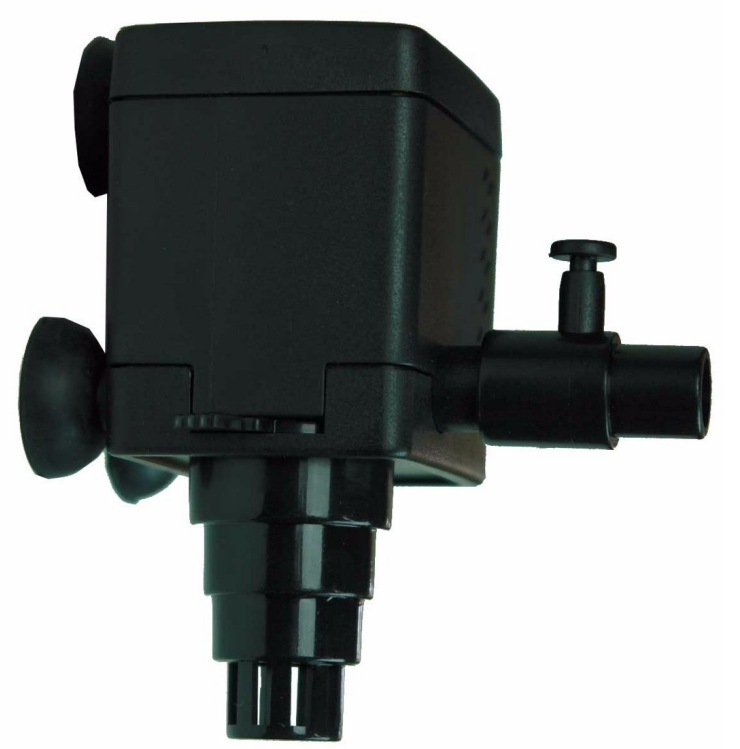

Figura 5. Moto Bomba de SB 1000 
- Circuito A Figura 6 mostra o modelo esquemático do projeto e nela pode-se verificar como o protótipo foi desenvolvido. Na esquematização do circuito observar-se que os componentes estão interligados à placa do microcontrolador ESP8266 em seu módulo de pinagem digital. Foram ligados no microcontrolador, o Modulo relé e o Sensor de Temperatura e Umidade DHT11, estando estes, por sua vez, conectados a um pino VCC de saída de energia de 3v, distribuída pela placa, e a um pino GND, de densidade neutra. A moto Bomba está ligada a um pino digital do Módulo Relé e a uma fonte bivolt 110/220v

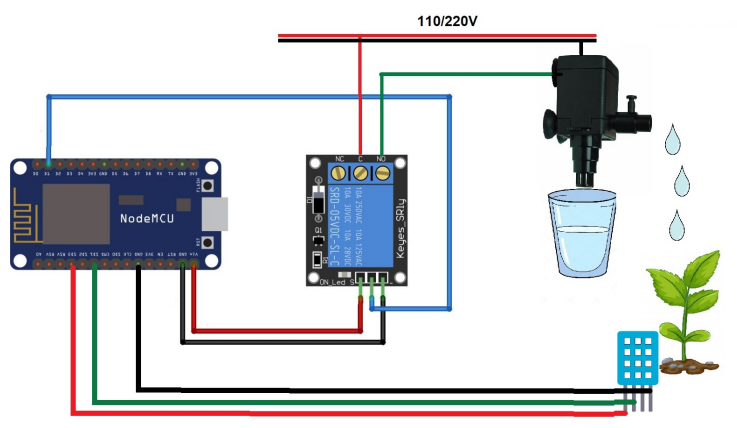

Figura 6. Esquema do Projeto

- Funcionamento A ativação do sistema de irrigação pode ocorrer de duas maneiras: na primeira, o usuário pré-estabelece uma porcentagem de umidade, de tal modo que, quando o sensor ler um dado de umidade menor ou igual ao determinado, a bomba de agoamento é acionada automaticamente; já no segundo modo, são disponibilizados, através de um webservice com um interface gráfica simples alocado no próprio microcontrolador Esp8266, com dois botões que fornecem as funções de acionar e interromper o sistema de agoamento, uma consulta por mês e ano das leituras armazenadas, e um campo de parâmetro para a umidade mínima que se deseja para que o sistema seja acionado. Essa cobertura de irrigação, tanto pelo parâmetro de umidade pré-definido quanto pelo acionamento em tempo real, é extremamente vantajosa para os grupos de pesquisadores, uma vez que permite um acompanhamento constante dos dados dos sistemas vegetativos cultivados, bem como garante que o agoamento será feito sempre que necessário, independente da presença humana no local. Por se tratar de um sistema desenvolvido para ambiente acadêmico, um grande diferencial disponibilizado pela ferramenta é o armazenamento de todos os dados de temperatura e umidade coletados através do sensor, tendo sido projetado, para este fim, um banco de dados em linguagem Mysql. Para fins de consulta temporais a essa base de dados, foi desenvolvida uma página web, que fornece aos pesquisadores do núcleo de estudos, NEAMB, a possibilidade de explorar os dados de uma maneira simplificada e em rede, tornandose extremamente útil para ampliação do horizonte de suas pesquisas.

\section{Resultados}

Para avaliar o protótipo foram realizados três testes:

- Manutenção da umidade em um percentual mínimo estabelecido pelo usuário;

- Monitoramento da temperatura; 
- Análise sobre a variação do nível de água da bomba.

Foi possível observar também, além do acompanhamento sobre a variação dos valores lidos pelos sensores, o armazenamento e as consultas à base de dados. Todos os testes foram realizados ao longo de um mês e com base nesses testes, foi possível aferir a efetividade do protótipo. Conforme constatado, é cabível afirmar que o mecanismo mostrou-se satisfatório, pois a realização desses três testes evidenciou o fato de que o protótipo se comportou conforme esperado e que o sistema foi capaz de oferecer ao usuário o valor da umidade e temperatura do solo em condições pré-determinadas e dentro de uma precisão suficiente para a aplicação de plantios. Adicionalmente aos testes específicos para funcionamento da ferramenta, foi realizado um último experimento, o qual objetivava validar o acionamento do sistema de irrigação pelo modo em rede, ou seja, atestar se o controle da válvula de água seria, de fato, acionado ao ser pressionado o botão "Ligar" conforme a Figura 7, apresenta a tela do aplicativo móvel. A confirmação de tal operação foi feita visualmente no protótipo, que demonstrou um funcionamento adequado da ativação do regador e dentro de um tempo de resposta aceitável.

\begin{tabular}{|c|c|c|}
\hline mês/ano & Buscar & \\
\hline Ligar & Desligar & \\
\hline Umidade \% & $\operatorname{Temp}^{\circ} \mathrm{C}$ & Data/Hora \\
\hline 84.00 & 28.00 & $12 / 12 / 2018$ 07:13:21 \\
\hline 84.00 & 28.00 & $12 / 12 / 201807: 13: 52$ \\
\hline 83.00 & 27.00 & $12 / 12 / 2018$ 07:14:22 \\
\hline 83.00 & 27.00 & $12 / 12 / 201807: 14: 53$ \\
\hline 83.00 & 27.00 & $12 / 12 / 2018$ 07:15:23 \\
\hline 83.00 & 27.00 & $12 / 12 / 201807: 15: 54$ \\
\hline 83.00 & 27.00 & $12 / 12 / 201807: 16: 24$ \\
\hline 63.00 & 26.00 & $12 / 12 / 2018$ 16:07:44 \\
\hline 58.00 & 27.00 & 12/12/2018 16:09:14 \\
\hline 58.00 & 27.00 & $12 / 12 / 2018 \quad 16: 09: 44$ \\
\hline 59.00 & 27.00 & $12 / 12 / 2018$ 16:10:40 \\
\hline 59.00 & 27.00 & $12 / 12 / 2018 \quad 16: 12: 59$ \\
\hline 59.00 & 27.00 & $12 / 12 / 2018$ 16:13:30 \\
\hline
\end{tabular}

Figura 7. Tela do Protótipo

\section{Discussões}

Pôde-se perceber através do desenvolvimento deste projeto onde o monitoramento dos padrões de umidade e temperatura do solo é uma ferramenta com grande potencial de ajuda na pesquisa da casa de vegetação de porto nacional e outros ambientes que possam auxiliar pesquisadores e promover um controle de recursos hídricos com custos relativamente baixos em relação ao que são encontrados no mercado atual. Hoje o protótipo está sento aplicado no NEAMB, em 18 mudas de milho, onde estão acontecendo pesquisas no desenvolvimento da espécie, com diversas técnicas de irrigação e fornecendo uma base de dados acessíveis de qualquer dispositivo com acesso à internet. $\mathrm{O}$ uso de internet das coisas viabiliza o monitoramento remoto do solo e abre espaço para desenvolvimento de novas pesquisas. Futuramente pretendemos utilizar uma versão mais robusta, com equipamentos profissionais para trabalhar nas lavouras respeitando suas especificações de cada espécie plantada, onde o produtor poderá estar em outro ambiente e monitorando sua lavoura. 


\section{Referências}

de Oliveira, S. (2017). Internet das coisas com ESP8266, Arduíno e Raspberry Pi. Novatec Editora.

dos Santos, S. and Pereira, G. M. (2010). Comportamento da alfalce tipo americana sob diferentes tensoes da agua no solo, em ambiente protegido. Disertacao (Maestrado em Irrigacao e Drenagem), Universidades Federal de Lavras, Brasil.

Júnior, J. B. C., Pereira, A., Stone, L., Aluísio, J., Moreira, A., and Klar, A. E. (2009). Efeito de métodos de determinação de parâmetros para o controle da irrigação na eficência do uso da água do feijoeiro, sob três diferentes lâminas de água no solo effects of parameter determination methods for common bean irrigation control. IRRIGAUNESP.

Menezes, J. d., Santos, T., Montenegro, A., and Silva, J. (2013). Comportamento temporal da umidade do solo sob caatinga e solo descoberto na bacia experimental do jatobá, pernambuco. Water Resources and Irrigation Management, 2(1):45-51.

Morgan, J. (2014 (accessed December 3, 2018)). A Simple Explanation of 'The Internet of Things.

Santos, B. P., Silva, L., Celes, C., Borges, J. B., Neto, B. S. P., Vieira, M. A. M., Vieira, L. F. M., Goussevskaia, O. N., and Loureiro, A. (2016). Internet das coisas: da teoria à prática. Minicursos SBRC-Simpósio Brasileiro de Redes de Computadores e Sistemas Distribuidos.

Souza, C. F., Matsura, E. E., et al. (2002). Avaliação de sondas de tdr multi-haste segmentadas para estimativa da umidade do solo. Revista Brasileira de Engenharia Agrícola e Ambiental. 